\title{
Clinical and neurophysiological spectrum of polyneuropathies in children
}

\author{
Elina Millere $^{1,2}$, Laura Gribuste ${ }^{2}$, Inese Kazaine ${ }^{1}$, Jurgis Strautmanis ${ }^{1,2}$, Linda Gailite ${ }^{2}$, \\ Viktorija Kenina ${ }^{1,2,3}$ \\ ${ }^{1}$ Children's Clinical University Hospital, Riga, Latvia \\ ${ }^{2}$ Riga Stradins University, Riga, Latvia \\ ${ }^{3}$ Riga East Clinical University Hospital, Riga, Latvia
}

\begin{abstract}
Peripheral neuropathy is a disorder of the peripheral nerves and results from a disturbance of structure and/or function of the peripheral sensory, motor and/or autonomic neurons. The possible aetiology of peripheral neuropathies is diverse, but inflammatory and hereditary diseases of the peripheral nerves predominate in childhood. The aim of this study was to determine the clinical and electrophysiological profile of large nerve fibre neuropathy detected by nerve conduction studies (NCS) in children over a 10-year period at the Children's Clinical University Hospital in Latvia. Based on NCS findings, 165 children between 2008 and 2018 were diagnosed with polyneuropathy. In our study, the majority of children had peripheral neuropathy due to acquired causes, mostly due to diabetes mellitus; roughly one in five of the patients had hereditary neuropathy. Almost half of the patients had motor deficits, which were more prevalent in toxic and inflammatory neuropathies. A little less than a third of patients complained of pain as well as presenting with autonomic dysfunction symptoms. The NCS demonstrated a demyelinating neuropathy in 52 cases (31\%), an axonal neuropathy in 34 cases (21\%), and mixed polyneuropathy in 79 cases (48\%). Our study investigated the clinical and electrophysiological characteristics of polyneuropathies diagnosed with NCS in children. Most of the polyneuropathies in our study were hereditary and diabetic neuropathies with combined (myelin and axon) damage to nerve fibres. Almost all clinical symptoms of polyneuropathy were present in all aetiological groups.
\end{abstract}

Key words: polyneuropathy, childhood, clinical, neurophysiology

(Neurol Neurochir Pol 2020; 54 (5): 466-470)

\section{Introduction}

Peripheral neuropathy is a disorder of the peripheral nerves and results from a disturbance of structure and/or function of the peripheral sensory, motor and/or autonomic neurons. Typically, the involvement of motor fibres can cause weakness, fasciculations, or muscle atrophy, whereas sensory involvement causes numbness, tingling, and/or altered perception of pain [1].

The possible aetiology of peripheral neuropathies is diverse and includes infection, autoimmune, neoplasmatic, endocrine, metabolic, congenital and toxic disorders. Among adult patients, metabolic polyneuropathies are the most frequent. However, inflammatory and hereditary diseases of the peripheral nerves predominate in childhood $[1,2]$.
The aim of this study was to determine the clinical and electrophysiological profile of large nerve fibere neuropathy detected by nerve conduction studies (NCS) in children over a 10-year period at the Children's Clinical University Hospital in Riga, Latvia.

\section{Methods}

Study participants

Study participants were enrolled in a tertiary hospital in Riga, Latvia. The study included children (i.e. under the age of 18 years) with polyneuropathy confirmed by NCS at the Children's Clinical University Hospital from 2008 to 2018. 


\section{Clinical and neurophysiological examination}

Medical history anamnesis, clinical and laboratory data were gathered from the patients' medical records. We retrospectively studied the results of all nerve conduction studies (NCS) performed between 2008 and 2018. NCS were performed in the sural nerve and at least one other motor nerve in each leg, and in two sensory nerves and one motor nerve in each hand. Two trained neurophysiologists independently re-evaluated the data from all nerve conduction studies.

The summarised data was broken down into groups based on clinical presentation or aetiology as having either hereditary or acquired causes. Acquired causes were categorised into being either diabetic, toxic, autoimmune, or neuropathy due to other cause.

\section{Ethics}

This study was approved by the the Ethics Committees of the Children's Clinical University Hospital and of Riga Stradins University (No. 4/22.02.2018).

\section{Results}

Based on NCS findings, 165 children between 2008 and 2018 were diagnosed with polyneuropathy. There was a slight male predominance: $55.8 \%(n=92)$ males and $44.2 \%(n=73)$ females. Patients were aged from one month to 17 years. The mean age was $12.25 \pm 5.10$ years.

The majority of children had peripheral neuropathy due to acquired causes (Tab. 1), mostly due to diabetes mellitus, followed by toxic causes. Almost all children with toxic polyneuropathy had been treated with chemotherapy. However, about one fifth of the patients had an inherited cause of peripheral neuropathy, and $64 \%(n=23)$ of them had Charcot Marie Tooth subtype 1A. However, in 15\% the cause of neuropathy was idiopathic. Inflammatory neuropathy patients had Guillain-Barre syndrome or chronic inflammatory polyneuropathy. Neuropathy due to other causes was due to Prader-Willi syndrome, coeliac disease, Waardenburg syndrome, neuroborreliosis, hereditary spastic paraparesis, 3-methylglutaric aciduria, mitochondrial disease, rheumatic diseases, or liver cirrhosis. In the patient group aged younger than 17 years $(\mathrm{n}=$ 112), the most prevalent cause for neuropathy was hereditary, followed by toxic causes.

Clinical features were summarised according to data from neurological examinations and medical records (Tab. 2). Almost half of the patients had motor deficits, which were more prevalent in toxic and inflammatory neuropathies. A little less than a third of patients complained of pain as well as presenting with autonomic dysfunction symptoms. In this group, the majority presented either toxic or diabetic causes of neuropathy. Sensory deficits were also more prevalent in toxic and diabetic neuropathies. Features of autonomic dysfunction included tachycardia $(n=22)$, gastrointestinal disturbances $(n=18)$, hypohidrosis $(n=6)$, hyperhidrosis $(n=4)$, and orthostatic hypotension $(\mathrm{n}=3)$. Almost all clinically mentioned symptoms were present in all aetiological subgroups of polyneuropathy.

The NCS in our patient group $(\mathrm{n}=165)$ demonstrated a demyelinating neuropathy in 52 cases (31\%), an axonal neuropathy in 34 cases (21\%), and mixed polyneuropathy in 79 cases $(48 \%)$. Demyelinating neuropathy was defined when nerve conduction velocity was decreased and/or nerve distal latency was prolonged; axonal neuropathy was defined when action potential amplitude was decreased; and mixed neuropathy was defined when both demyelinating and axonal neuropathy features were present.

A detailed NCS summary for a 126 patient group is set out in Table 3. Based on peripheral nervous system development and approximate maturation at 5-6 years [3], in NCS summary we excluded patients $0-6$ years of age $(n=30)$ and patients with neuropathy due to another disease ( $\mathrm{n}=11$, two of them aged under six). In the patient group $0-6$ years $(\mathrm{n}=30)$, NCS was evaluated individually according to age-specific NCS values and a diagnosis of neuropathy was made. Although patients with hereditary neuropathies can present with various NCS findings, our data showed prolonged latency with decreased conduction velocity, indicating the demyelination of peripheral nerves. The amplitude of the action potential was generally preserved. In cases of inflammatory neuropathy, patients tended to have similar findings as the hereditary neuropathy group, but with less severely expressed signs of demyelination.

Table 1. Aetiological spectrum of polyneuropathies

\begin{tabular}{lccc}
\hline \multicolumn{1}{c}{ Aetiology } & Number of patients (\%) & Number of patients aged < 17 (\%) \\
\hline Hereditary cause & $36(21.8)$ & $29(25.9)$ \\
Acquired cause & & $9(8.0)$ \\
& Diabetic & $40(24.2)$ & $25(22.3)$ \\
& Toxic & $31(18.8)$ & $20(17.9)$ \\
Autoimmune & $22(13.3)$ & $10(8.9)$ \\
Idiopathic & Due to other disease & $11(6.7)$ & $19(17.0)$ \\
Total & & $25(15.2)$ & $112(100)$
\end{tabular}


Table 2. Clinical features in polyneuropathies

\begin{tabular}{|c|c|c|c|}
\hline Clinical feature & & Number of patients (\%) & Total number of patients (\%) \\
\hline \multirow[t]{7}{*}{ Motor deficit } & & & $71(48.0)$ \\
\hline & Hereditary & $10(14.1)$ & \\
\hline & Inflammatory & $16(22.5)$ & \\
\hline & Diabetic & $1(1.4)$ & \\
\hline & Toxic & $21(29.6)$ & \\
\hline & Idiopathic & $15(21.1)$ & \\
\hline & Due to other disease & $8(11.3)$ & \\
\hline \multirow[t]{7}{*}{ Pain } & & & $47(29.7)$ \\
\hline & Hereditary & $5(10.6)$ & \\
\hline & Inflammatory & $9(19.1)$ & \\
\hline & Diabetic & $11(23.4)$ & \\
\hline & Toxic & $15(31.9)$ & \\
\hline & Idiopathic & $5(10.6)$ & \\
\hline & Due to other disease & $2(4.3)$ & \\
\hline \multirow[t]{7}{*}{ Sensory deficit } & & & $26(16.9)$ \\
\hline & Hereditary & $2(7.7)$ & \\
\hline & Inflammatory & $6(23.1)$ & \\
\hline & Diabetic & $7(26.9)$ & \\
\hline & Toxic & $8(30.8)$ & \\
\hline & Idiopathic & $2(7.7)$ & \\
\hline & Due to other disease & $1(3.8)$ & \\
\hline \multirow[t]{7}{*}{ Autonomic dysfunction } & & & $45(30.4)$ \\
\hline & Hereditary & $3(6.7)$ & \\
\hline & Inflammatory & $5(11.1)$ & \\
\hline & Diabetic & $16(35.6)$ & \\
\hline & Toxic & $18(40.0)$ & \\
\hline & Idiopathic & $0(0)$ & \\
\hline & Due to other disease & $3(6.7)$ & \\
\hline
\end{tabular}

Patients with toxic and diabetic neuropathies tended to have mild large myelinated nerve fibre damage with signs of demyelination. More signs of axonal damage were evident in the group with idiopathic neuropathies. More severe neuropathy findings were seen in leg nerves compared to hand nerves during NCS.

\section{Discussion}

Hereditary causes have traditionally been considered the most common causes of neuropathy in children [2], but they were only the second commonest cause in our study. The majority of children in our study had diabetic polyneuropathy. A possible bias affecting these results is the age distribution in our patient group: almost a third of our patients was aged 17 years. However, in analysing patients younger than 17, the most prevalent cause for neuropathy was hereditary. The incidence of diabetes in childhood is increasing worldwide
$[4,5]$, and NCS are used as a screening method for polyneuropathy in patients with diabetes more commonly than in cases with other pathologies. However, we assume that the high prevalence in our study could also be associated with the socioeconomic conditions in Latvia that could have a negative impact on timely visits to the GP as well as the use of medications, treatment compliance, and regular blood sugar monitoring. Furthermore, an additional possible cause for this could be the long delay between the first complaint/symptom and the diagnosis of hereditary neuropathy. According to our research, the mean time from first symptoms to diagnosis in the hereditary neuropathy group is 13.9 years (Millere et al., unpublished data).

It should be noted that our study participants were patients with NCS-confirmed polyneuropathy, and that some of the aetiological groups may have been underrepresented. Clear anamnestic reasons plus clinical symptoms, for example sensory deficits after chemotherapy or a genetically 
Table 3. Nerve conduction study findings in aetiological groups

\begin{tabular}{|c|c|c|c|c|c|}
\hline & $\begin{array}{l}\text { Hereditary } \\
\qquad n=25\end{array}$ & $\begin{array}{c}\text { Inflammatory } \\
\qquad n=17\end{array}$ & $\begin{array}{c}\text { Diabetic } \\
n=40\end{array}$ & $\begin{array}{c}\text { Toxic } \\
n=24\end{array}$ & $\begin{array}{l}\text { Idiopathic } \\
n=20\end{array}$ \\
\hline \multicolumn{6}{|c|}{ Median nerve } \\
\hline $\operatorname{MNCV}(\mathrm{m} / \mathrm{s})$ & $34.42 \pm 15.11$ & $48.81 \pm 16.81$ & $49.83 \pm 10.61$ & $51.47 \pm 14.25$ & $54.74 \pm 12.90$ \\
\hline DML (ms) & $12.59 \pm 6.04$ & $9.09 \pm 3.48$ & $4.04 \pm 1.79$ & $3.73 \pm 5.18$ & $3.90 \pm 1.08$ \\
\hline CMAP (mV) & $4.05 \pm 3.03$ & $5.76 \pm 3.31$ & $7.24 \pm 3.51$ & $4.19 \pm 2.93$ & $3.79 \pm 2.52$ \\
\hline $\operatorname{SNCV}(\mathrm{m} / \mathrm{s})$ & $23.87 \pm 24.14$ & $47.76 \pm 19.75$ & $48.09 \pm 10.68$ & $49.77 \pm 13.21$ & $48.02 \pm 17.70$ \\
\hline $\operatorname{SNAP}(\mu \mathrm{V})$ & $13.27 \pm 20.73$ & $31.32 \pm 19.97$ & $23.34 \pm 11.91$ & $21.34 \pm 14.32$ & $40.65 \pm 20.68$ \\
\hline \multicolumn{6}{|l|}{ Ulnar nerve } \\
\hline $\operatorname{MNCV}(\mathrm{m} / \mathrm{s})$ & $33.34 \pm 10.01$ & $49.34 \pm 10.82$ & $49.83 \pm 10.61$ & $50.31 \pm 10.25$ & $50.74 \pm 12.90$ \\
\hline DML (ms) & $8.27 \pm 2.83$ & $6.09 \pm 2.25$ & $3.4 \pm 1.71$ & $3.13 \pm 3.18$ & $3.90 \pm 1.18$ \\
\hline CMAP (mV) & $4.45 \pm 3.25$ & $10.76 \pm 2.31$ & $6.21 \pm 2.23$ & $7.19 \pm 2.93$ & $5.79 \pm 2.52$ \\
\hline $\operatorname{SNCV}(\mathrm{m} / \mathrm{s})$ & $23 \pm 21.12$ & $48.16 \pm 9.34$ & $47.19 \pm 9.38$ & $48.67 \pm 3.41$ & $47.32 \pm 7.40$ \\
\hline $\operatorname{SNAP}(\mu \mathrm{V})$ & $9.27 \pm 10.23$ & $12.23 \pm 2.94$ & $7.48 \pm 1.11$ & $11.34 \pm 14.32$ & $20.35 \pm 10.18$ \\
\hline \multicolumn{6}{|l|}{ Tibial nerve } \\
\hline $\operatorname{MNCV}(\mathrm{m} / \mathrm{s})$ & $25.09 \pm 15.26$ & $44.91 \pm 10.79$ & $40.66 \pm 5.47$ & $42.23 \pm 11.28$ & $42.51 \pm 14.33$ \\
\hline DML (ms) & $19.92 \pm 13.18$ & $14.09 \pm 3.90$ & $14.44 \pm 2.03$ & $12.31 \pm 4.33$ & $11.53 \pm 4.37$ \\
\hline CMAP (mV) & $2.24 \pm 2.72$ & $4.50 \pm 4.30$ & $9.72 \pm 14.46$ & $4.58 \pm 4.10$ & $8.47 \pm 6.55$ \\
\hline \multicolumn{6}{|l|}{ Sural nerve } \\
\hline $\operatorname{SNCV}(\mathrm{m} / \mathrm{s})$ & $17.85 \pm 22.53$ & $44.20 \pm 16.68$ & $34.63 \pm 22.98$ & $41.28 \pm 20.69$ & $45.86 \pm 24.84$ \\
\hline $\operatorname{SNAP}(\mu \mathrm{V})$ & $3.55 \pm 5.83$ & $11.08 \pm 6.98$ & $5.74 \pm 5.29$ & $7.04 \pm 7.25$ & $10.07 \pm 7.18$ \\
\hline
\end{tabular}

confirmed and known hereditary neuropathy diagnosis in a family, are possible reasons for avoiding NCS. Therefore, toxic and hereditary polyneuropathies may be underrepresented in our study.

The patient with Prader-Willi syndrome was nine months old with diffuse hypotonia when NCS was performed and the diagnosis of sensory demyelinating polyneuropathy was made. Although there is little evidence for neuropathy in Prader-Willi syndrome, we included this patient in the 'neuropathy due to other causes' group. It is possible that there could be another metabolic or hereditary cause for neuropathy in this patient, who needs to be monitored in the future.

Motor and sensory deficit, pain and autonomic dysfunction were present in almost all aetiological groups of polyneuropathies. Sensory symptoms are often under-recognised, especially in younger children [6], and our data also shows a sensory symptom burden in only $16.9 \%$ of patients. Diabetic and toxic neuropathy symptoms with prevalent sensory deficits, pain and autonomic dysfunction correspond to small nerve fibre damage, although we did not perform quantitative sensory testing (QST).

Although we considered hereditary neuropathies as one group, hereditary neuropathies are very heterogenous in clinical as well as NCS findings, either with more prevalent demyelination or axonal damage [7]. In Guillain-Barre syndrome, demyelination is the most prevalent neurophysiological finding [8], and our data also showed signs of demyelination in NCS.

Hereditary neuropathy complaints and early diagnosis is important for quality of life and early rehabilitation. For the most common hereditary neuropathy (Charcot Marie Tooth subtype 1A), therapy could be available soon [9]. In patients with diabetes, therapeutic efficacy and blood sugar control can be evaluated by neuropathy severity, and decisions on therapy corrections can be made accordingly.

Although NCS can be an unpleasant procedure for patients, it is a useful investigation to evaluate therapeutic success in diabetic neuropathy. It is a cheaper and faster method where genetic neuropathy is suspected compared to genetic testing.

\section{Conclusions}

Our study investigated the clinical and electrophysiological characteristics of polyneuropathies diagnosed with NCS in children. Most of the polyneuropathies in the present study were hereditary and diabetic neuropathies with combined (myelin and axon) damage to nerve fibres. Patients with hereditary neuropathies tended to have more severe changes and signs of demyelination in NCS, followed by inflammatory neuropathies, toxic and diabetic neuropathies. The latter 
tended to have milder signs of peripheral nerve fibre damage. Almost all clinical symptoms of polyneuropathy were present in all aetiological groups.

Funding: This research received no external funding.

Conflicts of Interest: The authors declare no conflict of interest.

\section{References}

1. Sommer C, Geber C, Young P, et al. Polyneuropathies. Deutsches Aerzteblatt Online. 2018, doi: 10.3238/arztebl.2018.0083.

2. Shabo G, Pasman JW, van Alfen N, et al. The spectrum of polyneuropathies in childhood detected with electromyography. Pediatr Neurol. 2007; 36(6): 393-396, doi: 10.1016/j.pediatrneurol.2007.02.013, indexed in Pubmed: 17560501.

3. Guchhait D, Sen D, Ganguly D, et al. Peripheral Motor and Sensory nerve conduction studies in normal infants and children in Eastern India. IOSR Journal of Dental and Medical Sciences. 2014; 13(8): 26-32, doi: 10.9790/0853-13832632.

4. Candler TP, Mahmoud O, Lynn RM, et al. Continuing rise of Type 2 diabetes incidence in children and young people in the UK. Diabet
Med. 2018; 35(6): 737-744, doi: 10.1111/dme.13609, indexed in Pubmed: 29460341.

5. Dabelea D, Mayer-Davis EJ, Saydah S, et al. SEARCH for Diabetes in Youth Study. Prevalence of type 1 and type 2 diabetes among children and adolescents from 2001 to 2009. JAMA. 2014; 311(17): 1778-1786, doi: 10.1001/jama.2014.3201, indexed in Pubmed: 24794371.

6. Wilmshurst J. Diagnosis and management of pediatric peripheral neuropathies in resource-poor settings. Future Neurology. 2013; 8(2): 133-148, doi: 10.2217/fnl.12.97.

7. Bird TD. Charcot-Marie-Tooth ( CMT ) Hereditary Neuropathy Overview 1. Clinical Characteristics of Charcot-Marie-Tooth ( CMT ) Hereditary Neuropathy. ; 2019: 1-22.

8. Dimachkie MM, Barohn RJ. Guillain-Barré syndrome and variants. Neurol Clin. 2013; 31(2): 491-510, doi: 10.1016/j.ncl.2013.01.005, indexed in Pubmed: 23642721.

9. Attarian S, Vallat JM, Magy L, et al. An exploratory randomised double-blind and placebo-controlled phase 2 study of a combination of baclofen, naltrexone and sorbitol (PXT3003) in patients with Charcot-Marie-Tooth disease type 1A. Orphanet J Rare Dis. 2014; 9: 199, doi: 10.1186/s13023-014-0199-0, indexed in Pubmed: 25519680. 